\title{
ACCELERATOR RELATED BACKGROUNDS IN THE LHC FORWARD DETECTORS*
}

\author{
N.V. Mokhov ${ }^{\dagger}$, A.I. Drozhdin, I.L. Rakhno, FNAL, Batavia, IL 60510, USA \\ D. Macina, CERN, Geneva, CH-1211, Switzerland
}

\section{Abstract}

Detailed Monte Carlo simulations are performed on radiation environment in the LHC IP5 interaction region at the locations of the TOTEM Roman Pots proposed to detect particles produced at very small angles in the elastic scattering and diffraction dissociation processes at the LHC. Radiation loads on these detectors are calculated with the MARS 14 code both of the $p p$-collision origin and beam loss related (beam-gas and tails from collimators).

\section{INTRODUCTION}

The TOTEM Roman Pot (RP) detectors will measure the total $p p$-interaction cross section, elastic scattering, and diffractive processes at the LHC [1]. These detectors consist of tracking devices placed into special units mounted on the vacuum chamber of the accelerator in the very forward region of the IP5: at 145 to $220 \mathrm{~m}$ from the CMS detector. Radiation doses and background particle rates at the RP locations are fundamental inputs to their design. The TOTEM will run at a luminosity not to exceed $10^{33} \mathrm{~cm}^{-2} \mathrm{~s}^{-1}$, i.e., ten times lower than nominal. Results of comprehensive studies of energy deposition effects due to $p p$-collisions are described in Ref. [2]. Accelerator related background in the CMS detector was calculated in Ref. [3]. Here we present results of detailed calculations of radiation effects in the forward detectors due to both $p p$ collisions and beam loss for the extended model based on the current version 6.4 lattice [4]. Operational beam loss rates in the vicinity of the detectors are studied taking into account both realistic vacuum distribution and apertures in the entire machine.

\section{MODELING IN IP5}

A new detailed calculation model is based on the current LHC lattice, IP5 component description of Ref. [2], latest advanced version of the MARS14 code system [5], and upgraded STRUCT code [6]. The MARS model of a 300-m long section includes all the essential components from the IP5 through the first two MB dipoles (Fig. 1). The RP stations (Fig. 2) are located at 145, 149, 180, 220, and $220.28 \mathrm{~m}$ from the IP. The last detector, RP5, is placed immediately after RP4 and rolled by $90^{\circ}$ around the z-axis.

Calculations are performed for the IP5(R) section for the $p p$-collisions at $10^{33} \mathrm{~cm}^{-2} \mathrm{~s}^{-1}$ with their products moving

\footnotetext{
* Work supported by the Universities Research Association, Inc., under contract DE-AC02-76CH03000 with the U.S. Department of Energy.

† mokhov@fnal.gov
}

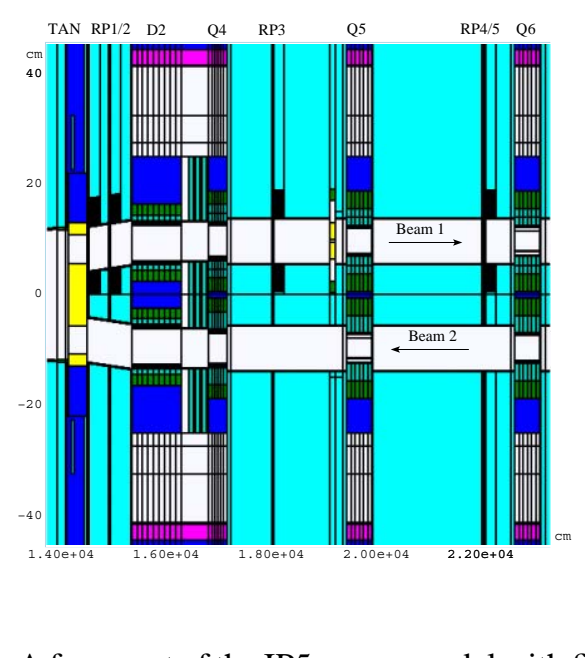

Figure 1: A fragment of the IP5 MARS model with five RPs.

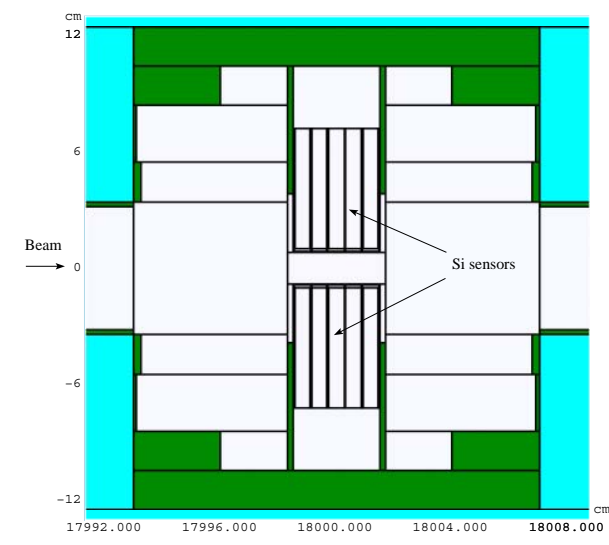

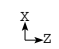

Figure 2: Elevation view of the RP MARS model.

in the Beam 1 direction. The TCL collimator in front of the Q5 quadrupole is in its garage position. Beam loss, calculated both for Beam 1 and 2, are induced by interactions of a $3.1 \times 10^{14}$ proton beams with collimators in the IP3 and IP7 and residual gas. A realistic vacuum distribution and both elastic and inelastic beam-gas interactions are taken into account. Note that a simplified pressure model and inelastic beam-gas interactions only were considered in Ref. [3]. The residual gas density distributions in strait warm and cold sections as well as in cryogenic arcs were recently estimated for the IP1 and IP5 [7]. The residual gas is represented as a mixture of four molecules $\left(\mathrm{H}_{2}, \mathrm{CH}_{4}\right.$, $\mathrm{CO}$, and $\mathrm{CO}_{2}$ ). The element concentration distribution in the IP5 is shown in Fig. 3. For the purpose of this study, 
the vacuum distribution in other regions is assumed to be the same as that in the IP5. A corresponding beam loss rate due to inelastic beam-gas interactions is presented in Fig. 4. The lowest pressure and, therefore, beam loss rate is observed in the warm sections. The value of estimated residual gas pressure in the warm sections of about $10^{-11}$ torr is unusually low when compared to that for other accelerators $\left(10^{-10}-10^{-9}\right.$ torr $)$. The main difference between the LHC warm sections and those for other machines is in a coating material for the vacuum chamber. For the LHC most of the warm vacuum chamber will be coated with TiZrV NEG. This coating has, after activation at low temperatures, a very high pumping speed for the components of the residual gas. Static pressures of about $10^{-13}$ torr were measured at laboratory conditions [7].

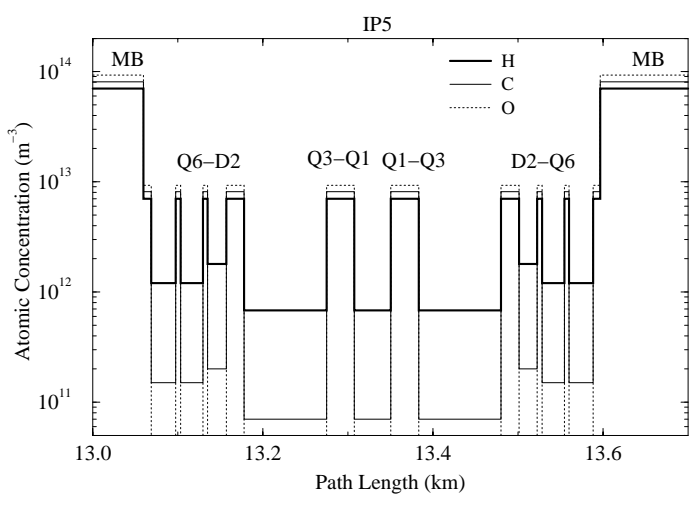

Figure 3: Residual gas concentration in the IP5.

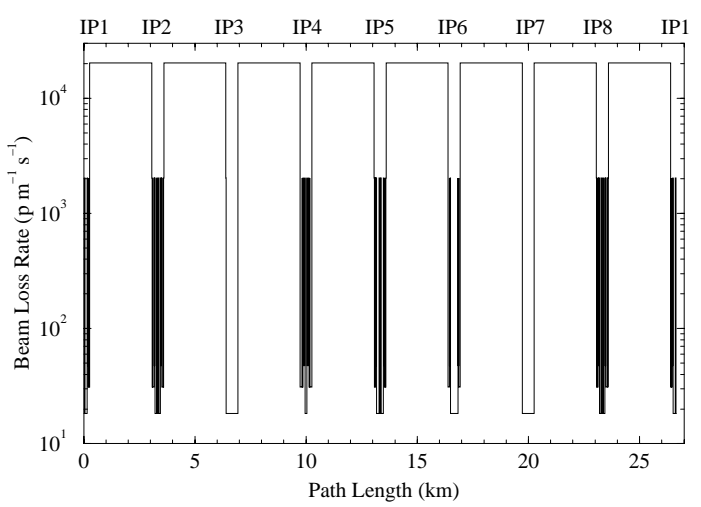

Figure 4: Estimated beam loss rate in the LHC lattice due to inelastic beam-gas interactions.

Beam loss due to the elastic beam-gas scattering [8] represents a "long-range" contribution in the region of interest because of very small scattering angles involved. On the contrary, inelastic beam-gas scattering gives rise to a "short-range" contribution. Therefore, calculation schemes for the two beam loss mechanisms are different. For elastic scattering, the first stage consists of a multi-turn STRUCT simulation of propagation of halo along with scattered protons $(\mathrm{E}>5 \mathrm{TeV})$ through the entire collider ring, similar to modeling the IP3 and IP7 collimator contribution. Scattered protons, lost on the LHC limiting apertures, form the source for the second stage - full shower simulation with the MARS code. Sample tracks of protons lost on the IP5 outer triplet apertures and picked up with MARS are shown in Fig. 5 for Beam 2. Most of the protons shown are lost on the Q4 and Q5 apertures and the tapered beam-pipe between the TAN and D1. For inelastic beam-gas interactions, large scattering angles are involved and, therefore, rather short regions (a few hundred meters) in the vicinity of the IP5 are considered in single-stage MARS runs.

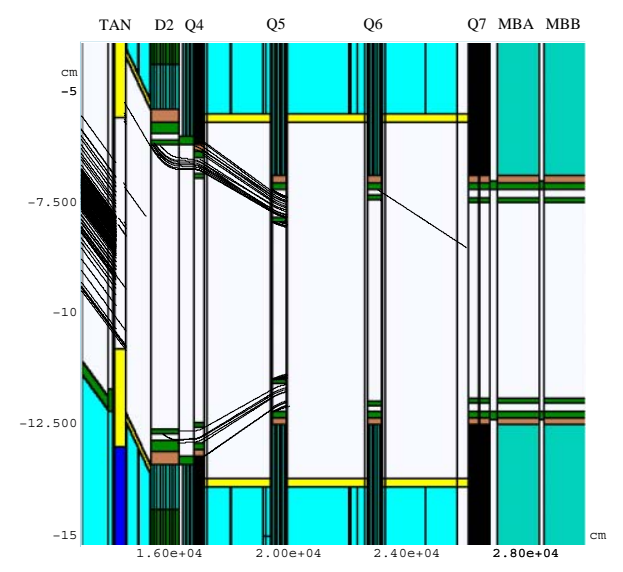

$\stackrel{\mathrm{Y}}{\rightarrow \mathrm{Z}}$

Figure 5: Sample tracks of the Beam 2 protons elastically scattered on residual gas around the ring and lost at $\mathrm{z}=140$ $270 \mathrm{~m}$ from the IP5.

\section{RADIATION LOADS IN TOTEM}

At normal operation conditions and nominal luminosity, the radiation resulting from colliding beam interactions dominates over all other sources. However, beam loss induced effects may play a role at a reduced luminosity. Fig. 6 shows beam loss distributions in the IP5 region calculated with STRUCT for Beam 1 interactions with the IP3 collimators [3] and residual gas (elastic) around the ring. The rates in the RP vicinity are of the order of hundredth of W/m (on L-side) and are comparable for the two sources. The MARS calculated dynamic heat loads to the separation dipole and outer triplet quadrupoles on the Rside are shown in Fig. 7 for $p p$-collisions at $10 \%$ of the nominal luminosity (Beam 1) and beam-gas elastic and inelastic interactions for Beam 2 (contribution from Beam 1 is negligible here). One sees that at the TOTEM conditions, $p p$-collisions are still the dominant source of radiation, although at some spots the total accelerator related load is comparable. A relatively small contribution of the beamgas inelastic component here can be understood if one takes into account a very high vacuum (low pressure) in this short region (dominant for inelastic interactions) compared to the rest of the machine starting at the MB dipoles (elastic interactions) as shown in Fig. 3.

Full-scale MARS simulations of showers induced in the TOTEM region are performed for all the sources described 


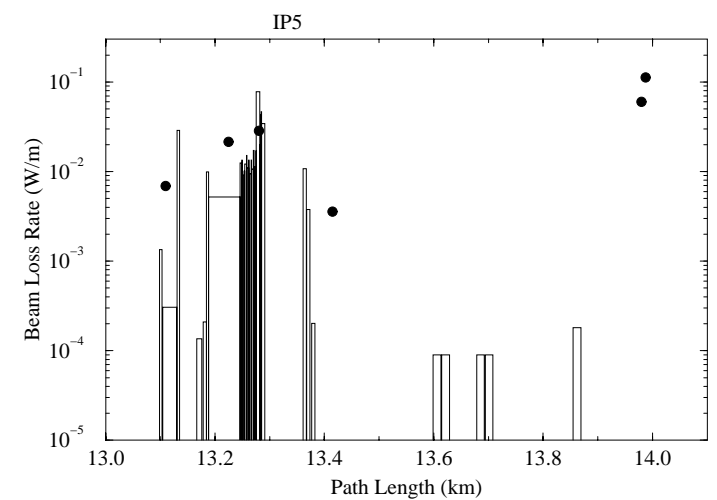

Figure 6: Beam loss rate in the IP5 caused by tails from collimators (symbols) and beam-gas elastic scattering (histogram).

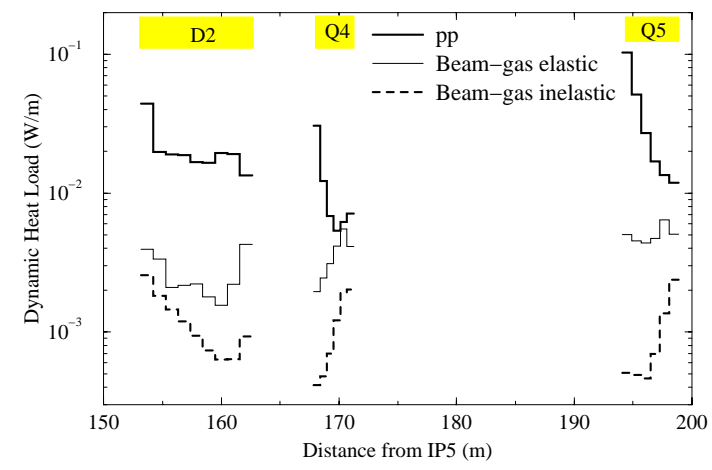

Figure 7: Dynamic heat load on the IP5(R) D2, Q4 and Q5 magnets due to $p p$-collisions at $10^{33} \mathrm{~cm}^{-2} \mathrm{~s}^{-1}$ and beamgas elastic and inelastic interactions.

above. Calculated are particle fluxes above $0.1 \mathrm{MeV}$, absorbed dose, energy and angular distributions at the RP silicon sensors as well as radiation loads to accelerator and other components at larger radii in this region. The results revealed that the $p p$-collisions dominate significantly even at the TOTEM luminosity of $10^{33} \mathrm{~cm}^{-2} \mathrm{~s}^{-1}$. Backgrounds induced by tails from the collimators and beam-gas elastic and inelastic scattering are of the order of $0.1-1 \%$ of the total rates at the RPs. An example of a 2D distribution of neutron flux is shown in Fig. 8 for the RP5. Table 1 gives the particle fluxes and absorbed dose on the silicon sensors of all the RP detectors calculated at $10^{33} \mathrm{~cm}^{-2} \mathrm{~s}^{-1}$. Also shown is a distance $\mathrm{d}_{S i}=10 \sigma+0.5 \mathrm{~mm}$ between the inner edge of the RP sensor and beam axis.

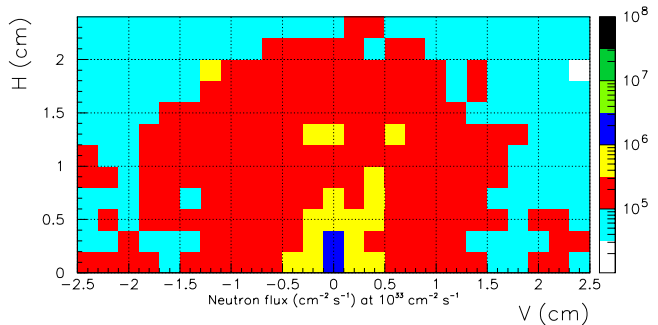

Figure 8: Total neutron flux isocontours averaged over the six RP5 silicon sensors.
Table 1: Total particle fluxes $\left(10^{5} \mathrm{~cm}^{-2} \mathrm{~s}^{-1}\right)$ averaged over silicon sensors and peak and average absorbed doses $\left(10^{4} \mathrm{~Gy} / \mathrm{yr}\right)$.

\begin{tabular}{|l|c|c|c|c|c|}
\hline Item & RP1 & RP2 & RP3 & RP4 & RP5 \\
\hline Charged & & & & & \\
hadrons & 7.5 & 2.9 & 1.0 & 0.58 & 6.7 \\
Neutrons & 3.7 & 1.3 & 0.61 & 0.37 & 1.4 \\
Electrons & 115 & 28 & 23 & 12 & 18 \\
Photons & 1410 & 279 & 187 & 81 & 147 \\
\hline $\mathrm{D}_{\max }$ & 12 & 10 & 20 & 1.9 & 55 \\
$\mathrm{D}_{a v}$ & 3.4 & 1.1 & 0.69 & 0.37 & 1.2 \\
\hline $\mathrm{d}_{S i}$ & 10.36 & 10.33 & 8.77 & 5.97 & 2.10 \\
\hline
\end{tabular}

\section{CONCLUSIONS}

Detailed calculations performed for the TOTEM Roman Pots in the IP5 region with the up-to-date lattice, detector parameters, MARS model, and realistic vacuum distribution reveal the high background rates on the silicon sensors, exceeding $10^{12} \mathrm{~cm}^{-2}$ of charged hadrons per year with the peak dose of about $100 \mathrm{kGy} / \mathrm{yr}$. We found that the $p p$-collisions dominate even at $1 / 10$ of the nominal luminosity, with backgrounds induced by tails from the collimators and beam-gas elastic and inelastic scattering contributing $0.1-1 \%$ to the above values.

Authors thank J.-B. Jeanneret and A. Rossi of CERN for information on the LHC apertures and newest vacuum model.

\section{REFERENCES}

[1] G. Matthiae, TOTEM Technical Proposal, CERN/LHCC 997 (1999).

[2] N.V. Mokhov, I.L. Rakhno, J. S. Kerby, J. B. Strait, "Protecting LHC IP1/IP5 Components Against Radiation Resulting from Colliding Beam Interactions", Fermilab-FN-732 (2003); LHC Project Report 633, CERN, Geneva (2003).

[3] A.I. Drozhdin, M. Huhtinen, N.V. Mokhov, Nucl. Instr. and Methods in Phys. Research, A 381 (1996), 531-544.

[4] http://proj-lhc-optics-web.web.cern.ch/proj-lhc-optics-web/V6.4/.

[5] N. V. Mokhov, "The MARS Code System User's Guide", Fermilab-FN-628 (1995); N.V. Mokhov, "Status of MARS Code", Fermilab-Conf-03/053 (2003); http://www-ap.fnal.gov/MARS/.

[6] I.S. Baishev, A.I. Drozhdin, N.V. Mokhov, "STRUCT Program User's Reference Manual", SSCL-MAN0034 (1994); http://www-ap.fnal.gov/ drozhdin/.

[7] A. Rossi, N. Hilleret, CERN Memo, March 2003.

[8] A.I. Drozhdin, V.A. Lebedev, N.V. Mokhov et al., Fermilab-FN-734 (2003). 